\title{
Study on the pitching motion model of a high-speed monohull ship based on test
}

\author{
Zou Ze $\mathrm{e}^{1, \mathrm{a},{ }^{*}}$, Cao zhiwei ${ }^{1, \mathrm{~b}}$,Yang Songlin ${ }^{2, \mathrm{c}}$ \\ ${ }^{1,2,3}$ College of Marine and Shipbuilding Engineering,Jiangsu University of Science and Technology, Mengxi \\ Road,Zhenjiang, Jiangsu, China \\ a zouze_jkd@163.com, b 897118610@qq.com, cysl560516@vip.163.com
}

Keywords: high-speed monohull ship; pitching motion model; hydrodynamic coefficients; system identification; genetic algorithms

\begin{abstract}
In this paper, the author took a type of high-speed monohull ship as the research object, designed and added a bulbous bow of original ship by the principle of best comprehensive performance, made two 2.7 meters long scale models. Then conducted a series of pitch decay tests on condition that two ship models were in different drafts and tilt angles, established a mathematical model by the method of system identification based on genetic algorithms, developed a system identification software, made an analysis on pitching motion mode. The pitch motion differential equations of the two models in different drafts and initial tilt angles and the changing regularities of hydrodynamic coefficients with draft and initial tilt angle were obtained and comparatively analyzed. Through the research, the method and results can provide technical support to the deeper analysis and designs of this kind of ship.
\end{abstract}

\section{Introduction}

As observed by statistical data, when the ship sailing on the sea, it will be affected by the different sizes and directions of wave forces in $70 \%$ of the time. So it is difficult to avoid the oscillation of the ship in various angles, such as hanging, pitching, rolling.Among them, the pitching is an very important part of the ship's rocking movement.It is also self-evident that the significant influence of ship's manoeuvrability and navigation safety in the wave ${ }^{[1]}$. When the ship occur pitching under the influence of waves,and it usually results in the increase of the ship's navigation resistance, the ship speed loss, the slamming at the bottom of the ship, green water and propeller emergence and so on.These factors will cause baneful influence on the navigational performance directly or indirectly of the ship.At the same time, it brings difficulties to ship handling,and even lead to some serious consequences such as ships out of control and shipwrecks.It poses a serious threat to the safety of ships sailing in the sea.Therefore, as the main part of the evaluation of ship navigation performance, increase the study of the seakeeping of ships in oscillation motion in wave environment, and the practical application to guarantee safety of navigation of ships in waves, are one of the hot topics in the research of ship oscillation motion performance $^{[2-4]}$.

Pitch motion is often accompanied by the heave and roll motion, it usually lead to the complex hydrodynamic characteristics of the ship ${ }^{[5]}$.The theoretical prediction of pitch motion is still the weak link in the seakeeping theory. The damping moment coefficients determined by purely theoretical methods can not be applied in reality. At present, the method of system identification is used to identify the hydrodynamic derivatives of the maneuvering motion of ships, and establishing mathematical model to predict the short-term movement of the maneuvering and control of the maneuvering motion ${ }^{[6-7]}$.

This paper established a mathematical model by the method of system identification based on genetic algorithm,developed a system identification software, to verify the reliability of system identification, obtained the pitch motion differential equations of the two models in different drafts and initial tilt angles and comparatively analyzed the changing regularities of hydrodynamic coefficients with draft and initial tilt angle. 


\section{Experiment models and experiment scheme}

\subsection{Experiment models}

In this paper, a high-speed single hull ship is taken as the object of study.A bulbous bow was designed and fitted to the prototype ship, and make a scale model of $2.63 \mathrm{~m}$ without bulbous bow and $2.73 \mathrm{~m}$ with bulbous bow.

Table 2.1 Main scale parameters of prototype ship model

\begin{tabular}{ccccc}
\hline No. & Design variable name & Symbol & Unit & Size \\
\hline 1 & Length & $L$ & $\mathrm{~m}$ & 2.63 \\
2 & Width & $B$ & $\mathrm{~m}$ & 0.23 \\
3 & Moulded depth & $T 1$ & $\mathrm{~m}$ & 0.14 \\
4 & Designed draft & $T 2$ & $\mathrm{~m}$ & 0.083 \\
5 & Mid-ship section coefficient & $C_{m}$ & & 0.877 \\
6 & Block coefficient & $C_{b}$ & & 0.536 \\
\hline
\end{tabular}

Table 2.2 Parameters of bulbous bow model

\begin{tabular}{ccccc}
\hline No. & Design variable name & Symbol & Unit & Size \\
\hline 1 & Relative length of protrusion & $\mathrm{lb} / \mathrm{lbp}$ & & 0.044 \\
2 & Relative submergence & $h b / T$ & & 0.614 \\
3 & Maximum width ratio & $b m a x / B$ & & 0.212 \\
4 & Relative displacement ratio & $\delta / \Delta$ & & 0.028 \\
\hline
\end{tabular}

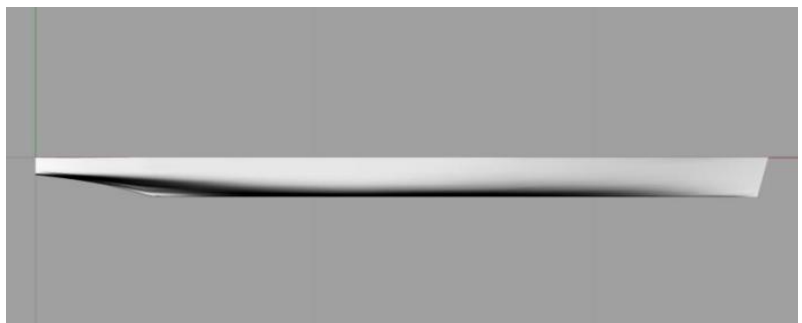

Figure 2.1 the original ship model

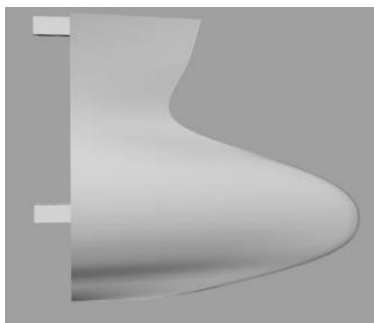

Figure 2.2 installing bulbous bow model

\subsection{Experimental equipment}

MTi-G is a miniature inertial measurement system integrated GPS. It exports three-axis acceleration,angular velocity and attitude angle (pitch / roll / yaw). Anti vibration gyroscope chip built-in high precision (10 degrees/h) can export high precision three axis attitude angle in vibration environment and non-uniform magnetic field.

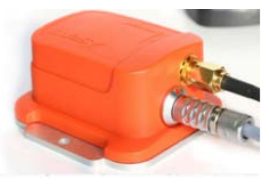

Figure 2.3 Mti-G

\subsection{Experiment contents}

The experimental was conducted in the wave tank of Jiangsu University of Science And Technology.Put the Mti-G in centre-of-gravity position of ship model.

(1)Laying up the model without bulb bow in the water tank. When the ship model is stable, loading the ballast and make sure the ship model is floating on even keel, then read the initial angle of the ship in the still water;

(2)Pressing with external force on the ship model in designed draft and make ship model incline at $1^{\circ}, 2^{\circ}, 3^{\circ}$, then releasing the external forces and collecting data;

(3)Loading $5 \%$ of standard displacement of ballast and repeat step(2);

(4)Continue loading 5\% of standard displacement of ballast and repeat step(2);

(5)Replace another ship with bulb bow, and repeat step (1) (4). 


\section{Identification model and method of pitching motion}

\subsection{System identification}

Figure 3.1 is the schematic diagram of the system identification.The input data in system identification is $U(\mathrm{k})$, the output data is $\mathrm{Y}(\mathrm{k})$, the process of system identification by $\mathrm{P}$, a mathematical model represented by $M, L$ and $C$ respectively represent optimization algorithms and criteria for system identification.The difference between the system output data $\mathrm{Y}(\mathrm{k})$ and the model output data $\mathrm{Y}_{\mathrm{M}}(\mathrm{k})$ is the output error $\varepsilon(k)$.Through the optimization algorithm of $\mathrm{L}$ and criteria $\mathrm{C}$ to make the output error criterion $\varepsilon(k)$ tends to zero.The corresponding identification parameters obtained can make the mathematical model $\mathrm{M}$ and the system identification $\mathrm{P}$ process to be the best.And then complete the process of constructing mathematical model by using system identification method.

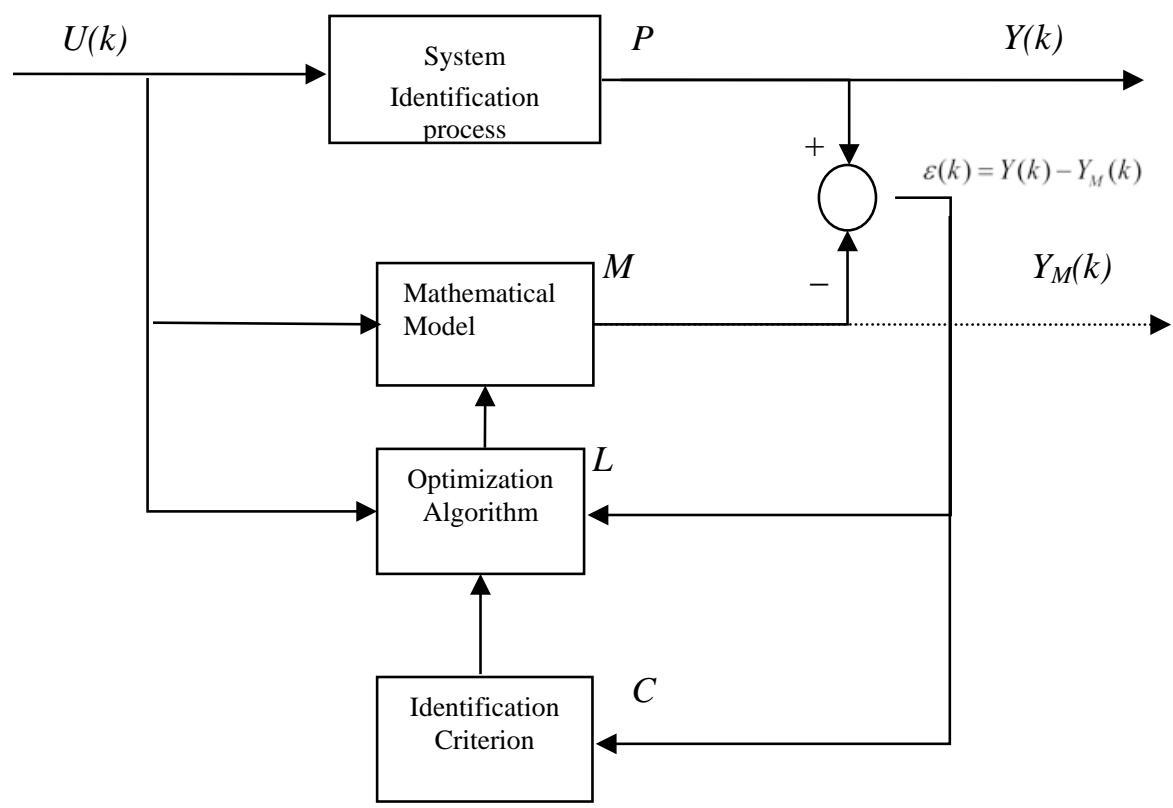

Figure 3.1 The principle of system identification

In this paper, the off-line identification algorithm is used, and the implementation process of system identification is shown in Figure 3.2. 


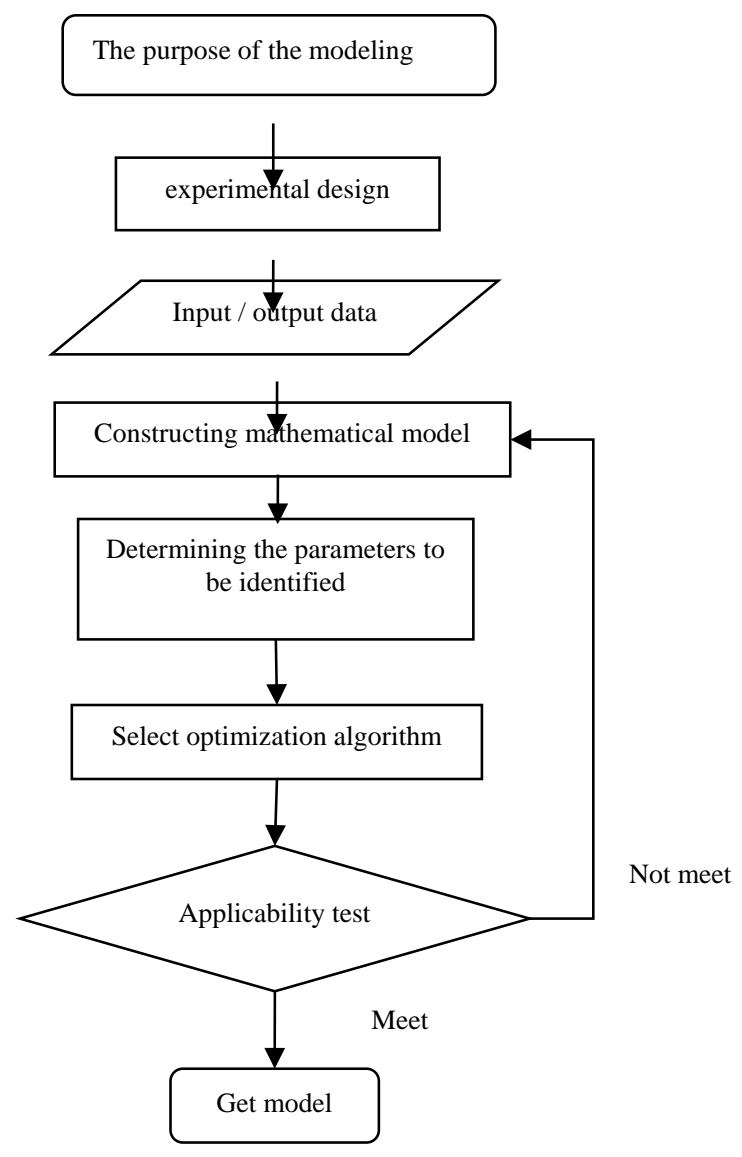

Figure 3.2 Implementation process of system identification

\subsection{Mathematical model}

The following equation of pitching motion is established when the sum of the moments of the $\mathrm{x}$-axis is zero and the ship motion and the force characteristics are taken into account:

$$
\ddot{\varphi}+2 \frac{N_{\theta}}{I_{X}} \dot{\varphi}+\frac{W}{I_{X}}|\dot{\varphi}| \dot{\varphi}+\frac{x}{I_{X}} \dot{\varphi}^{3}+\frac{C_{1}}{I_{X}} \varphi=0
$$

Where: $I_{x}$ — the total pitch moment of inertia of the hull; $\varphi$ —pitch angle $\dot{\varphi}$-pitch velocity; $\ddot{\varphi}$ —pitch angular acceleration; $N_{\theta}$ — the linear damping coefficient of the pitch; $\mathrm{W}, \mathrm{x}$ — the nolinear damping coefficients of the pitch; $\mathrm{C}_{1}$ - the linear righting moment coefficient;

Predict the pitch angle velocity at $\mathrm{K}+1$ moment:

$$
\dot{\varphi}_{K+1}=\left(-2 \frac{N_{\theta} t}{I_{X}}+1\right) \dot{\varphi}_{K}-\frac{W t}{I_{X}}\left|\dot{\varphi}_{K}\right| \dot{\varphi}_{K}-\frac{x t}{I_{X}} \dot{\varphi}_{K}^{3}-\left(\frac{\Delta \mathrm{h}}{I_{X}} \varphi_{k}\right) t
$$

By testing the pitching angle velocity at the time of $\mathrm{K}$, then we have the error criterion at the time of $\mathrm{K}+1$ :

$$
\varepsilon_{k+1}=\varphi_{k+1}^{\prime}-\varphi_{k+1}
$$

Where: $\varphi_{k+1}^{\prime}$ is the measured values of angle for the $\mathrm{k}+1$ moment.

Then we got the fundamental formula with estimated optimum condition (called loss function):

$$
J_{k+1}=\left|\varepsilon_{k+1}\right|
$$


The objective function is: $F(x)=\sum_{k=1}^{N} J_{k+1}^{2}$

Choose $I_{Y}, N_{\varphi}, W, \mathrm{x}, C_{1}$ as the design variables, and then we set

$I_{\mathrm{x}} \in(0,1) W \in(0,1) N_{\varphi} \in(0,1)$, constraint condition and objective functions constitute the standard mathematical model of optimization.

The optimization algorithm uses the parallel genetic algorithm, that is the design variables to be divided in parallel within the upper and lower bounds of the design variables, and a genetic algorithm is used to optimize the algorithm in each sub region.

Use the recognition software of pitch which I have written and the user interface is shown in Figure 3.3.The number of initial population was set as 200, the number of iterations was 4000 , the cross was 0.8 and mutation rate was 0.15 .

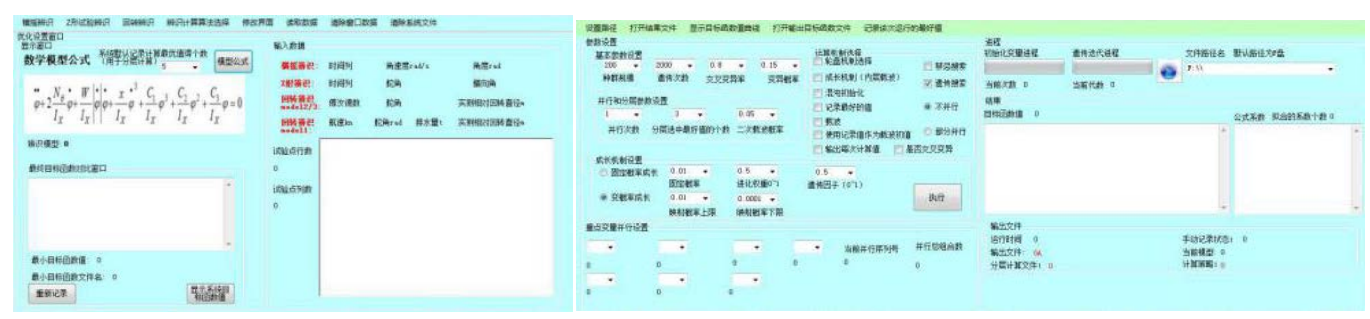

Figure3.3 Identifying software interfaces

The restoring torque term in pitching motion equation is changed from linear to nonlinear, and the equation is :

$$
\ddot{\varphi}+2 \frac{N_{\theta}}{I_{X}} \dot{\varphi}+\frac{W}{I_{X}}|\dot{\varphi}| \dot{\varphi}+\frac{x}{I_{X}} \dot{\varphi}^{3}+\frac{C_{1}}{I_{X}} \varphi^{3}+\frac{C_{2}}{I_{X}} \varphi^{2}+\frac{C_{3}}{I_{X}} \varphi=0
$$

Where: $I_{x}$-the total pitch moment of inertia of the hull; $\varphi$ _pitch angle $\dot{\varphi}$-pitch velocity; $\ddot{\varphi}$ —pitch angular acceleration; $N_{\theta}$ — the linear damping coefficient of the pitch; $\mathrm{W}, \mathrm{x}$ - the nonlinear damping coefficients of the pitch; $C_{1}, C_{2}$ - the nonlinear restoring moment coefficients , $C_{3}$ the nonlinear restoring moment coefficients. Using the same method to discrete and identification.

\section{Analysis of test results}

Based on the above identification mathematical model and optimization method, the calculation software is authored.

4.1 Comparing two sets of data of the two models in the designed draft and the initial pitch angle of $1^{\circ}$ and $3^{\circ}$,results are as follows:

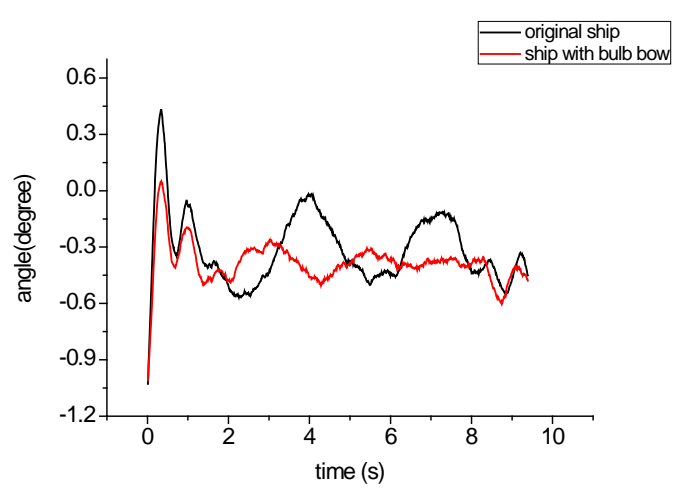

(a) $1^{\circ}$

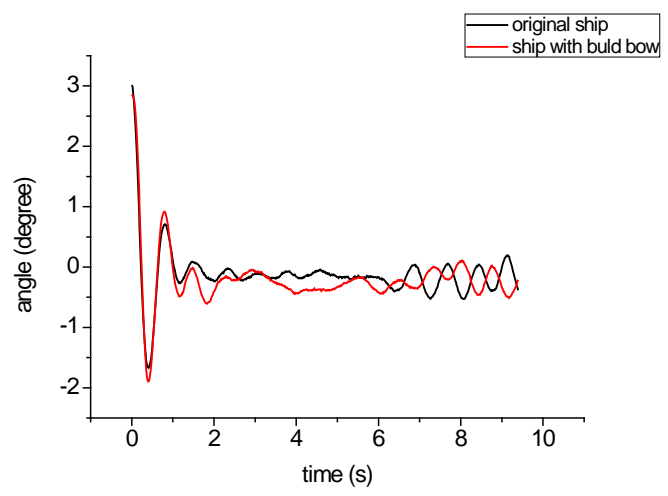

(b) $3^{\circ}$

Figure 4.1 The decay curve of pitching angle in designed draft 
Under the condition of small angle, a ship with bulbous bow model pitching amplitude is much smaller than another ship without bulbous bow.The bulbous bow has obvious anti-rolling effect.In the condition of large angle, it also has a faint effect.

4.2 Analyzed the results of the identification of the dynamic equilibrium equations of linear and nonlinear restoring torque terms of the bulbous bow ship model under the standard draft with the initial pitching angles of $1^{\circ}, 2^{\circ}$ and $3^{\circ}$.

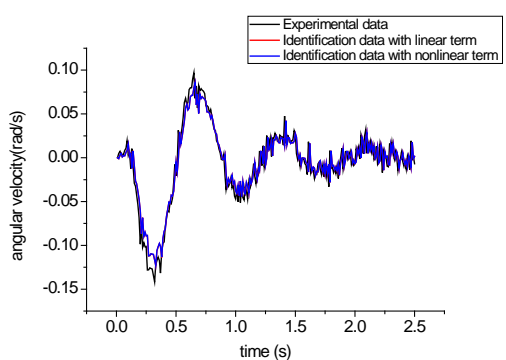

(a) $1^{\circ}$

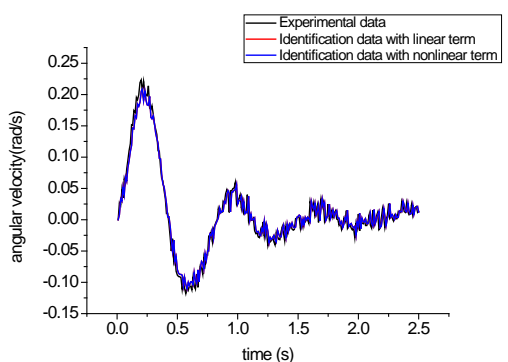

(b) $2^{\circ}$

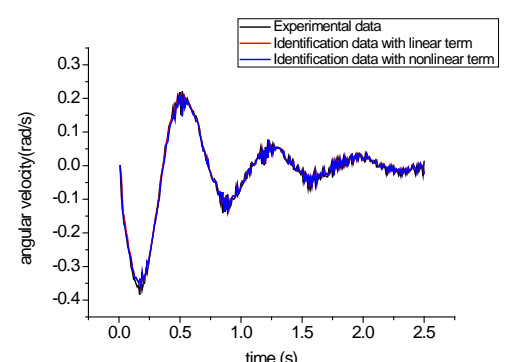

(c) $3^{\circ}$

Figure 4.2 The fitting decay curves of pitch angular velocity

As it can be seen from the figure,the identification data of three angles and test data are good.At the condition of $1^{\circ}$ and $2^{\circ}$,the linear and nonlinear identification data coincide basically, but at the condition of $3^{\circ}$,linear and nonlinear identification data have a little error.After the first cycle,the two sets of data basically coincide again. As the initial angle increased,the nonlinear objective function become more and more larger.At $1^{\circ}$ and $2^{\circ}$, the error tends to be zero,and at $3^{\circ}$ the error reaches $0.603 \%$.We can find that the two equations can basically describe the state of ship model motion in the case of small angle.As the angle increased the pitching motion equation with nonlinear restoring moment is better.

4.3Selected identification results of the model without bulbous bow in designed draft and initial pitch angle of $2^{\circ}(\mathrm{A})$ and the original ship in $105 \%$ of designed displacement under the initial pitch angle of $3^{\circ}(\mathrm{B})$ with nonlinear restoring moment term for analysis.

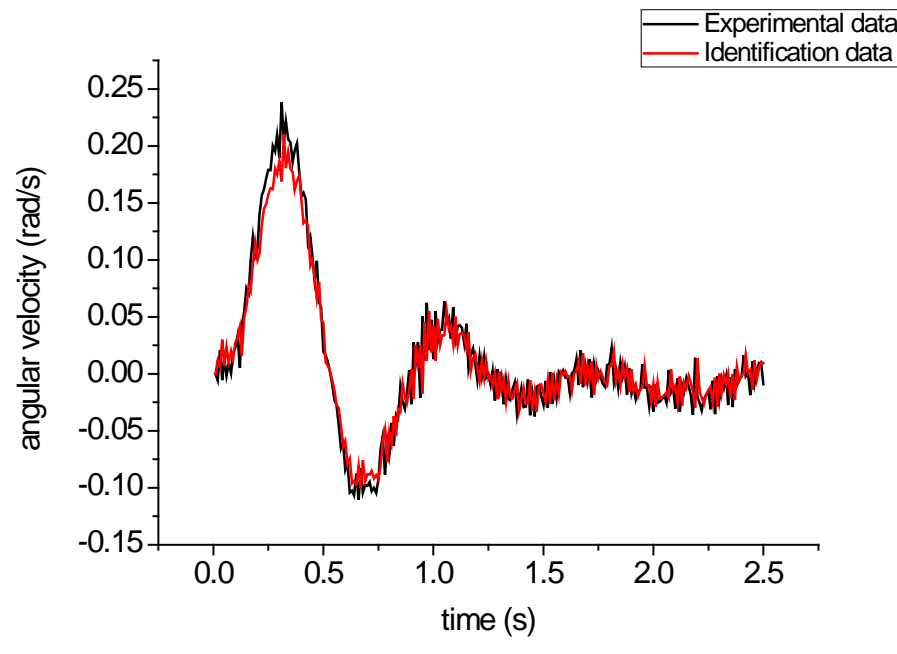

Figure 4.3 The decay curve of angular velocity(A) 
Table 4.2 Error of experimental and identification date

\begin{tabular}{cccc}
\hline Time(s) & $\begin{array}{c}\text { Experimental angular } \\
\text { velocity }\end{array}$ & $\begin{array}{c}\text { identification angular } \\
\text { velocity }\end{array}$ & Error(\%) \\
\hline 0.13 & 0.150840081 & 0.146249008 & 3.043669079 \\
0.14 & 0.170991652 & 0.166267329 & 2.762896767 \\
0.15 & 0.191556867 & 0.184652896 & 3.604136738 \\
0.16 & 0.19936547 & 0.180918224 & 9.252979679 \\
0.17 & 0.223379455 & 0.205239619 & 8.120637393 \\
0.18 & 0.204140691 & 0.208718431 & 2.242443679 \\
0.19 & 0.205154727 & 0.205553767 & 0.194506738 \\
0.2 & 0.198340962 & 0.190342666 & 4.032599075 \\
0.21 & 0.197943026 & 0.183564237 & 7.264104962 \\
0.22 & 0.17607056 & 0.163588652 & 7.089151199 \\
0.23 & 0.181884252 & 0.161118467 & 11.41703305 \\
0.24 & 0.168827444 & 0.166037903 & 1.65230322 \\
0.25 & 0.15205483 & 0.153567487 & 0.994810344 \\
0.26 & 0.144216556 & 0.137707625 & 4.513303534 \\
0.27 & 0.136182806 & 0.130115384 & 4.455351168 \\
\hline
\end{tabular}

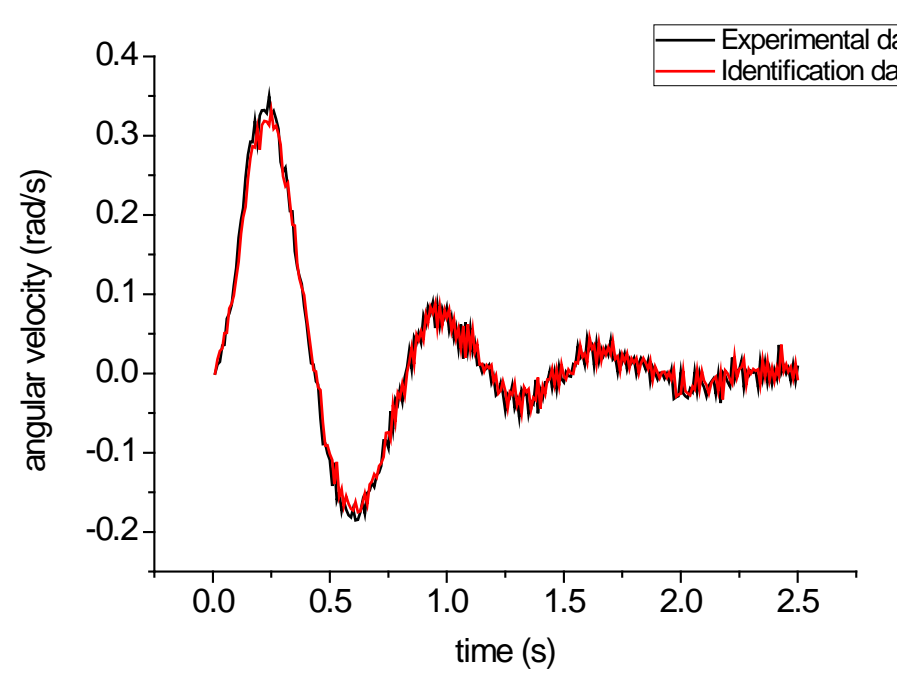

Figure 4.4 The decay curve of angular velocity(B)

Tab 4.3 Error of experimental and identification date

\begin{tabular}{cccc}
\hline Time(s) & $\begin{array}{c}\text { Experimental angular } \\
\text { velocity }\end{array}$ & $\begin{array}{c}\text { identification angular } \\
\text { velocity }\end{array}$ & Error(\%) \\
\hline 0.21 & 0.33204889 & 0.31336568 & 5.626644384 \\
0.22 & 0.332354323 & 0.318663656 & 4.11929855 \\
0.23 & 0.328579176 & 0.317886914 & 3.254090099 \\
0.24 & 0.349702896 & 0.313284594 & 10.41406942 \\
0.25 & 0.326074628 & 0.332039521 & 1.829302933 \\
0.26 & 0.331319343 & 0.308769994 & 6.805925941 \\
0.27 & 0.320796753 & 0.312646665 & 2.540576827 \\
0.28 & 0.308267034 & 0.301723636 & 2.122639689 \\
0.29 & 0.2668207 & 0.288952053 & 8.29446642 \\
0.3 & 0.254343341 & 0.249106376 & 2.059014054 \\
0.31 & 0.259696266 & 0.236554691 & 8.911015834 \\
0.32 & 0.233401136 & 0.240761437 & 3.153498518 \\
0.33 & 0.204927834 & 0.215274616 & 5.048987887 \\
0.34 & 0.204430415 & 0.187828084 & 8.121262718
\end{tabular}

Two kinds of pitching motion equations are: 


$$
\begin{aligned}
& \ddot{\varphi}+12.7 \dot{\varphi}+0.005|| \dot{\varphi} \mid \dot{\varphi}+0.002 \dot{\varphi}^{3}+107.8 \varphi^{3}+8.13 \varphi^{2}+33.8 \varphi=0 \\
& \ddot{\varphi}+5.89 \dot{\varphi}+0.001|\dot{\varphi}| \dot{\varphi}+0.08 \dot{\varphi}^{3}+118.4 \varphi^{3}+0.15 \varphi^{2}+31.9 \varphi=0
\end{aligned}
$$

We can find that the two curves are fitting good in the whole process.Comparatively speaking,the error in wave peak and trough is larger, others are quite small.

4.4The mathematical models with the linear restoring moment term of the original ship are obtained.Then comparing and analyzing the total pitching moment of inertia- $I_{X}$, Pitching linear damping torque coefficient $-N_{X}$, quadratic damping damping $-\mathrm{W}$, three square damping $-\mathrm{X}$ and coefficient of restoring moment- $\mathrm{h}$.

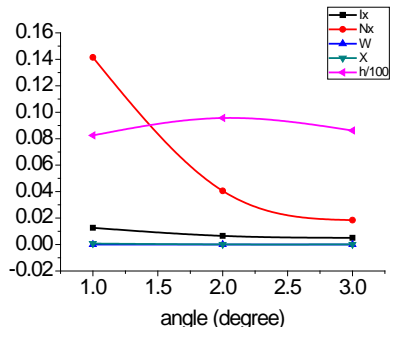

(a) $105 \%$

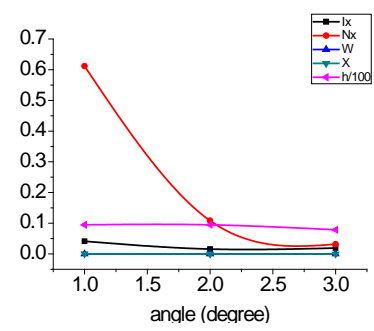

(b) $105 \%$

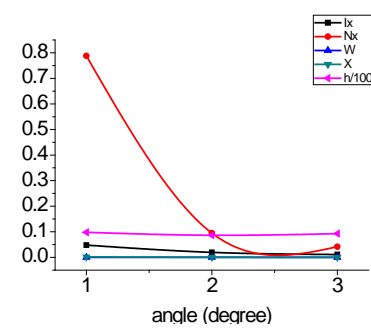

(c) $110 \%$

Figure4.5 The changes of the hydrodynamic coefficients in different drafts

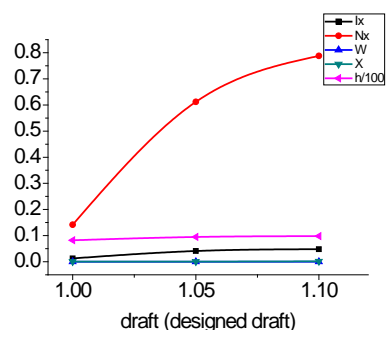

(a) $1^{\circ}$

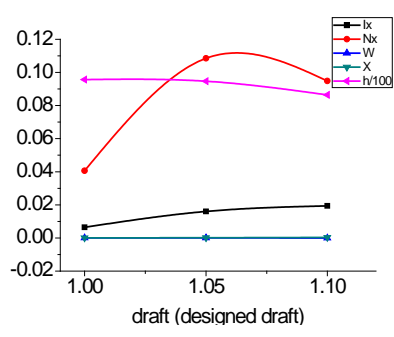

(b) $2^{\circ}$

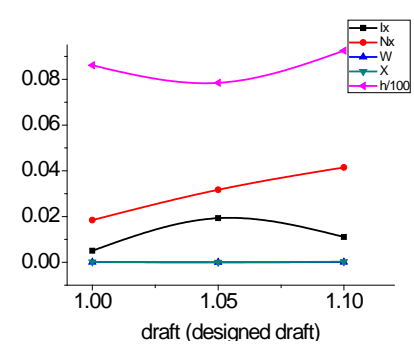

(c) $3^{\circ}$

Figure4.6 The changes of the hydrodynamic coefficients in different initial angles Using the same way to the ship model with bulbous bow:

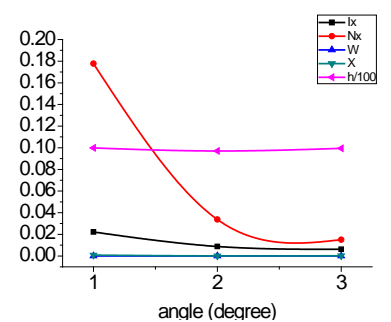

(a) $105 \%$

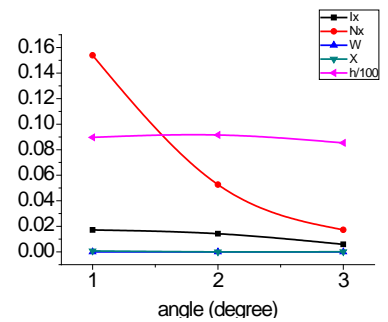

(b) $105 \%$

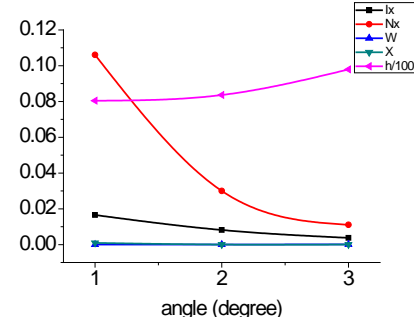

(c) $110 \%$

Figure4.7 The changes of the hydrodynamic coefficients in different drafts

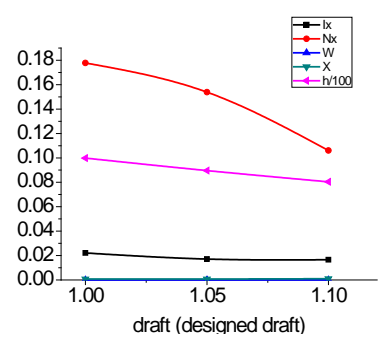

(a) $1^{\circ}$

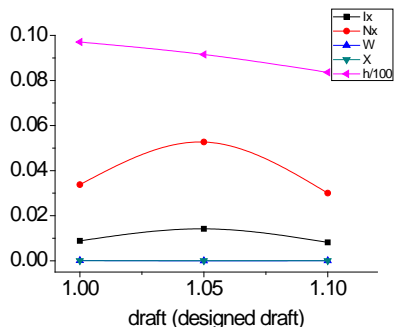

(b) $2^{\circ}$

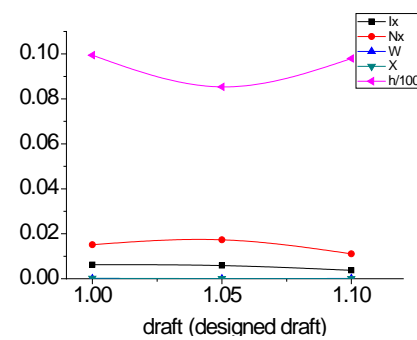

(c) $3^{\circ}$

Figure4.8 The changes of the hydrodynamic coefficients in different initial angles 
From figure4.5-4.8 we can find that,the values of the torque coefficients vary with the draft and the initial pitching angle.The total moment of inertia $I_{X}$ and the linear damping moment coefficient $N_{X}$ of the two ships are decreasing with the increase of the initial pitching angle.The square damping $W$ and the cubic damping $X$ are almost zero and remain stable.It is shown that the nonlinear damping is a tiny proportion in small angle of pitching motion. The restoring torque coefficient h of the two types of ships decreases with the increase of drafts at $1^{\circ}$ and $2^{\circ}$. But with the draft increased, it decreased at first and then increased at $3^{\circ}$.

\section{Analysis of the experiment results}

In this paper,the experiments of free damping of the pitch motion of two kinds of ship models are conducted,and obtained eighteen angular velocity changing curves of pitching motion in different pitching angles and drafts. The author reprogramed the system identification program which is based on Visual Basic 6.0 and used the mathematical model of nonlinear pitching attenuation motion as a variable.The curves of the angular velocity were fitted respectively.Analyzed the variation of hydrodynamic coefficients in different drafts and initial pitching angles and obtained the following conclusions:

(1) Compared with the experimental data and the identification results of genetic algorithm identification program which based on VB platform.The two sets of data are fitting good.The feasibility of the set of mathematical models and identification procedures is verified;

(2) When the initial angle is $1^{\circ}$ and $2^{\circ}$, the pitching motion equations with linear and nonlinear restoring torque can simulate the pitching state very well.the data identified by the two equations of motion are almost completely overlapping.At $3^{\circ}$ a slight error occurs. The pitching motion equation with nonlinear response moment in the large angle is better than the linear one.

(3) The bulbous bow has a certain effect on reducing the pitching of the ship model in small angles.The anti-rolling effect was even more obvious in the condition of small angles than the large angles.

(4) In the condition of small angles,the pitching only has linear damping basically.There is almost no nonlinear damping. The total moment of inertia $I_{X}$ and the linear damping torque coefficient $N_{X}$ are decreasing with the increase of the initial pitching angle.

\section{Reference}

[1] Yangshan Dai , Jinwei Shen , Jingzheng Song. Ship wave load[M].Beijing:National Defense Industry Press.2007.

[2] Guoze Xia, Gang Ma.Ship hydrodynamics[M].Wuhan:Huazhong University of Science and Technology Press.2003.

[3] Jide Li . Seakeeping[M].Harbin Engineering University Press.2007.

[4] ZHANG Yao, WANG Kai, YANG Shuai, et al. Hull lines optimization based on the evaluation of seakeeping performance [J] . Chinese Journal of Ship Research, 2015, 10(6):8-14.

[5] D Dumitru,Numerical investigation of a two - degrees - of - freedom ship model for pitch - roll motion [C] ,IOP Conference Series: 10.1088/1757-899X/145/8/082007,2016.

[6] Lennart ljung. System identification theory for the user[M].Tsinghua University Press, 2002.

[7] Chongzhi Fang , Deyun Xiao. Process identification[M]. Tsinghua University Press, 2003.

[8] Minghong She . Analysis and research of genetic algorithm applied in system identification

[J] .Research Findings, 2016, 11. 